\title{
Estratégia de interação on-line: um estudo no contexto de falantes da língua portuguesa de Portugal ${ }^{1}$
}

\section{On-line interaction strategy: A study in the context of the Portuguese language in Portugal}

\author{
Querte Mehlecke* \\ Liane Tarouco ${ }^{* *}$ \\ Margarete $\mathrm{Axt}^{* * *}$ \\ Alda Pereira \\ Janete Costa ${ }^{* * * * * *}$
}

\begin{abstract}
This article presents a research report carried out in the Department of Sciences and Education, DCE, at the Open University, Lisbon, Portugal. In this research it is shown an analysis of the textual messages submitted to ForChat, an on-line learning environment, during a time span of two months. The objective of the textual messages analysis was to identify the strategies of communication utilized by three lecturers who belong to the Distance Education and Educational Projects, PEED, on its stage 3.

Resumo. Este artigo apresenta um relato de pesquisa realizado no Departamento de Ciências da Educação, DCE, da Universidade Aberta, Lisboa, Portugal. Nesta pesquisa é apresentado a análise das mensagens textuais postadas no ForChat, ambiente de suporte à aprendizagem on-line durante o período de dois meses. O objetivo da análise das mensagens textuais foi o de identificar as estratégias de comunicação utilizada por três professores que fazem parte dos Projectos Educacionais e Ensino a Distância, PEED em sua fase 3.
\end{abstract}

\section{Introdução: Cenário de Estudo}

Esta pesquisa situa-se na confluência de estudos desenvolvidos no Programa de PósGraduação em Informática na Educação - PPGIE/UFRGS - nas seguintes linhas de pesquisa: Ambientes Informatizados e Ensino a Distância. O tema está relacionado ao estudo das estratégias de comunicação on-line. A pesquisa dá-se a partir de um estudo de caso, de natureza qualitativa, e analisa o conjunto das mensagens textuais postadas por professores num ambiente de suporte à aprendizagem on-line. Esta pesquisa se constituiu a partir do estágio de doutoramento, na Universidade Aberta, Portugal, com os professores do Departamento de Ciências da Educação (DCE).

\footnotetext{
${ }^{1}$ Trabalho extraído da tese apresentada no dia 30 de março de 2006. Programa de Pós-Graduação em Informática na Educação, PGIE, UFRGS. CAPES.

*FACCAT, Faculdades de Taquara, RS, Brasil; CINTED, PPGIE - Universidade Federal do Rio Grande do Sul (UFRGS), Caixa Postal 15.064 - 91.501-970 - Porto Alegre, RS, Brasil.

**CINTED, PGIE - Universidade Federal do Rio Grande do Sul (UFRGS).

***PGIE, LELIC - Universidade Federal do Rio Grande do Sul (UFRGS).

****Universidade Aberta, Lisboa, Portugal.

*****PGIE, CINTED - Universidade Federal do Rio Grande do Sul (UFRGS); FACCAT. 
A partir deste contexto, analisam-se as estratégias de comunicação/interação utilizadas por três professores, no ambiente de suporte à aprendizagem on-line, o ForChat.

Nesse contexto, cabe mencionar Axt e Elias (2003), que apresentam, como uma possibilidade de metodologia de trabalho, pode provocar, em ambientes educativos virtuais - e articuladamente aos estudos e questionamentos de ordem teórica e epistemológica - condições de possibilidade para encontros entre pensamentos, encontros esses que possam implicar seus participantes, de um modo muito especial, em experiências de aprendizagem de natureza cognitivo-conceitual e, ao mesmo tempo, ético-vivencial (p.260).

Os métodos e as técnicas utilizadas para o alcance dos objetivos propostos da pesquisa baseiam-se no dialogismo bakhtiniano, expresso nas mensagens textuais dos professores, postadas no ForChat. Nesse contexto, procura-se ancorar a metodologia construída para a análise das mensagens textuais, nas relações de diálogo, possibilitadas e apoiadas pelo ambiente de suporte à aprendizagem on-line - Forchat.

\section{Ambiente de Suporte à Aprendizagem On-line: ForChat}

O ForChat possibilita tanto a comunicação síncrona quanto assíncrona, mantendo o registro (das mensagens textuais) das interações durante o período de sua utilização. Durante esse período, professores e alunos podem retornar aos textos (mensagens postadas) anteriores, podendo interagir em qualquer parte dos diálogos. Essa opção possibilitada pelo ForChat é de fundamental importância, pois todos os registros de acesso dos alunos ficam arquivados. Assim, tem-se a possibilidade de buscar passagens já postadas e de retornar aos diálogos tantas vezes forem necessárias. A estrutura do ForChat baseia-se num complexo conceitual que busca privilegiar o acesso ao ambiente de modo mais intuitivo, ao compor, ou fundir, num mesmo espaço, simultaneamente, as funções de chat, fórum e mural, mantendo o usuário imerso no texto de leitura ao mesmo tempo em que ele se dedica a produzir seu próprio texto.

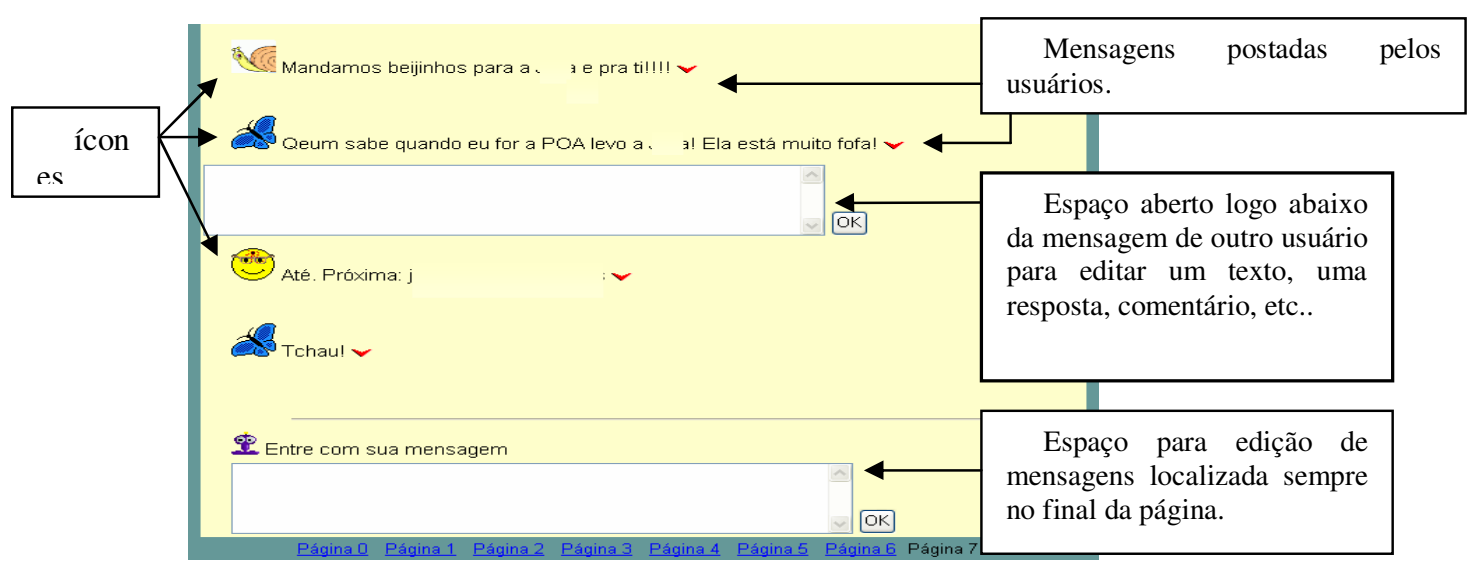

Figura 1: Tela de comunicação on-line: ForChat 
Segundo Axt $(2005)^{2}$, são seis os princípios tecno-metodológicos que dão sustentação ao ForChat:

1) o princípio da simplicidade;

2) o princípio da agilidade;

3) o princípio da imersão;

4) o princípio da liberdade;

5) o princípio da alteridade;

6) o princípio do desinstitucionalização de posições pré-instituídas.

A exposição dos princípios tecno-metodológicos do ForChat contribuiu para a contextualização da metodologia desta ferramenta que estava sendo utilizada pelos professores da Universidade Aberta. A análise dos dados se deu a partir das mensagens postadas pelos professores no Forchat, em três grupos do Seminário de Projetos Educativos na Universidade Aberta, Portugal, dos quais a autora desta pesquisa participou como membro da equipe de professores/pesquisadores ${ }^{3}$ que estavam a investigar as estratégias utilizadas pelos professores e alunos na modalidade a distância. Nos Seminários de Projetos Educativos foram acompanhadas e observadas as mensagens dos professores postadas no ForChat, no período de dois meses, para os alunos com o objetivo de identificar e descrever as estratégias utilizadas por eles. $\mathrm{O}$ contexto desses Seminários compôs e enriqueceu a pesquisa, pois deles pode-se fazer uma análise do conjunto de mensagens buscando identificar as estratégias de comunicação, produzidas pelos professores no ForChat.

\section{Análise do conjunto de mensagens textuais postadas pelos professores no ForChat}

A análise das mensagens textuais postadas pelos professores no ForChat aqui apresentada foi construída a partir da metodologia de análise fundada por Axt, em "Micro-agenciamentos de enunciações coletivas em comunidades virtuais de aprendizagem" e já utilizadas por Moreira, Souza e Ledur (2005).

Com base na metodologia construída por Axt, busca-se, nas mensagens textuais postadas no ForChat, os critérios atitudinais para definir as estratégias interacionais utilizadas pelos professores na comunicação on-line com seus alunos. Os critérios atitudinais criados para definir as estratégias interacionais levaram em conta o que se considerou a atitude de comunicação mais saliente do professor em relação ao aluno, contida no conjunto de mensagens postadas por ele no ForChat. Os critérios atitudinais criados pela pesquisadora para a análise dos dados são os indicadores para realizar o levantamento das estratégias interacionais utilizadas pelos professores do Seminário de Projetos Educativos.

De acordo com Alves-Mazzotti e Gewandsznajder (2004, p.171), os procedimentos utilizados na presente análise buscam maximizar sua confiabilidade segundo os critérios atitudinais definidos pelo contexto em foco. Assim, a análise qualitativa dos dados levantados (mensagens textuais dos professores) se traduz pela mediação dos critérios atitudinais e das estratégias interacionais. Ou seja, os critérios que norteiam os procedimentos de análise das estratégias interacionais, emergentes das mensagens textuais postadas pelos professores no ForChat, se configuram em torno das

\footnotetext{
${ }^{2}$ Palestra proferida pela Prof. ${ }^{\text {- Dr. }}{ }^{\text {a }}$ Margarete Axt, ao DCE, Universidade Aberta, Lisboa, Portugal, dia 20 de janeiro de 2005, em transmissão por videoconferência, do PPGIE/UFRGS, por ocasião do Workshop..

${ }^{3}$ Professores pesquisadores do DCE. V. $4 \mathrm{~N}^{\circ} 1$, Julho, 2006
} 
combinações entre as seguintes atitudes criadas para a identificação das estratégias: 1)Crítico/reflexivo; 2) Explicativo; 3)Fático/incentivo.

A partir da criação dos critérios atitudinais é que se dá a análise das mensagens textuais dos professores os quais servem para a identificação das estratégias interacionais utilizadas por eles. Para Van Dijk (1992), cada situação comunicacional/interacional pode requerer novas estratégias e a partir desse conceito é que se verifica, no decorrer das análises, as possíveis estratégias interacionais utilizadas pelos professores no ForChat.

A partir dos textos resultantes de diversas vozes, onde a voz do professor é pronunciada através das mensagens textuais, ele procura diferentes estratégias para movimentar os alunos para o diálogo/interação on-line.

No que diz respeito à forma escrita, os ambientes de suporte à aprendizagem on-line, propiciam espaços para a escrita individual ou coletiva. Pode-se dizer que uma das estratégias interacionais utilizadas nesses ambientes caracteriza-se pela atitude e estratégias interacionais, pois é através do(s) diálogo(s) que os interlocutores estabelecem formas comunicacionais entre os sujeitos que interagem e dialogam entre si. A interação, segundo Bakhtin (1992), é entendida como toda a comunicação verbal, de qualquer tipo que seja. Nesse sentido, é interessante salientar que as interações referidas neste estudo se dão nas mensagens textuais postadas no ForChat.

As estratégias selecionadas para esta análise configuram-se através das combinações de estratégias interacionais relacionadas a seguir:

- $\quad$ Enunciativa/diretiva - 1;

- Responsiva de caráter restrito/pergunta-resposta - 2;

- $\quad$ Dialógica -3 .

Para melhor compreensão e acompanhamento do processo de análise, cria-se um modelo de apresentação para cada conjunto de mensagens analisadas, em consonância com a seguinte codificação:

\begin{tabular}{|l|l|}
\hline Denominação & Codificação \\
\hline Critérios & Cr \\
\hline Estratégia & Est \\
\hline Professor & P \\
\hline Mensagem & M \\
\hline
\end{tabular}

Quadro 1: Denominação e codificação para as análises dos dados

Partindo dos códigos apresentados no quadro 1, o conjunto de mensagens analisadas será apresentado conforme o exemplo a seguir. Exemplo:

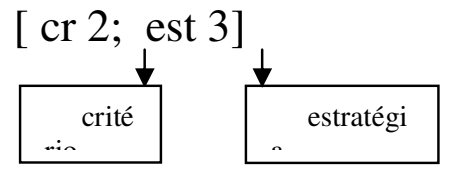

Figura 2: Exemplo da apresentação dos dados

Quanto às estratégias, em número de três, estas foram construídas a partir da posição de destaque nas mensagens textuais do professor frente às mensagens dos alunos, no ForChat, no contexto da dinâmica interacional: 1) estratégia enunciativa diretiva; 2) estratégia 
responsiva de caráter restrita; 3) estratégia dialógica. O quadro 2, a seguir, descreve os critérios e as estratégias selecionados para a análise.

\begin{tabular}{|l|l|l|l|}
\hline Critério = cr & Descrição & Estratégia = est & Descrição \\
\hline 1. Crítico/ reflexivo & $\begin{array}{l}\text { Intervenções críticas } \\
\text { Promove reflexões } \\
\text { Desenvolve } \\
\text { questionamentos }\end{array}$ & 1. Enunciativa Diretiva & $\begin{array}{l}\text { Em anúncios gerais sobre a } \\
\text { temática em estudo. }\end{array}$ \\
\hline 2. Explicativo & $\begin{array}{c}\text { Explica, orienta } \\
\text { Responde a questionamentos }\end{array}$ & $\begin{array}{l}\text { 2. Responsiva e de caráter } \\
\text { restrito; } \\
\text { pergunta-resposta. }\end{array}$ & $\begin{array}{l}\text { Em resposta às questões } \\
\text { feitas pelos alunos. } \\
\text { Monológico. }\end{array}$ \\
\hline 3. Fático $/$ Incentivo & $\begin{array}{l}\text { Poucas informações } \\
\text { Faz-se presente nondo acontece troca de } \\
\text { ambiente } \\
\text { Incentiva a participação }\end{array}$ & 3. Dialógica discussóes, reflexões \\
\hline
\end{tabular}

Quadro 2: Descrição geral dos critérios e das estratégias

Para a análise das mensagens observadas pela pesquisadora no ForChat, foram realizadas várias leituras do contexto do Seminário de Projetos Educativos - mensagens postadas pelos professores no ForChat - para que se pudesse chegar ao quadro 2, de apresentação geral dos critérios atitudinais e das estratégias interacionais observadas, em combinação livre.

O quadro 2 acima, apresenta os critérios atitudinais para definir as estratégias interacionais do conjunto de mensagens postadas pelos três professores no ForChat. A partir do quadro 2, selecionou-se e organizou-se grupos de mensagens postadas pelos três professores no ForChat, de acordo com o critério e a estratégia identificados nas mensagens.

Com base na identificação dos critérios atitudinais e estratégias interacionais, apresentase as análises das mensagens textuais postadas no ForChat pelos três professores. Observe a Figura 1 que ilustra as combinações de critérios e estratégias utilizadas pelo Professor 1, totalizando 213 mensagens no período de dois meses.

\section{Critérios (Cr) e estratégias (est) utilizadas pelo Professor 1}
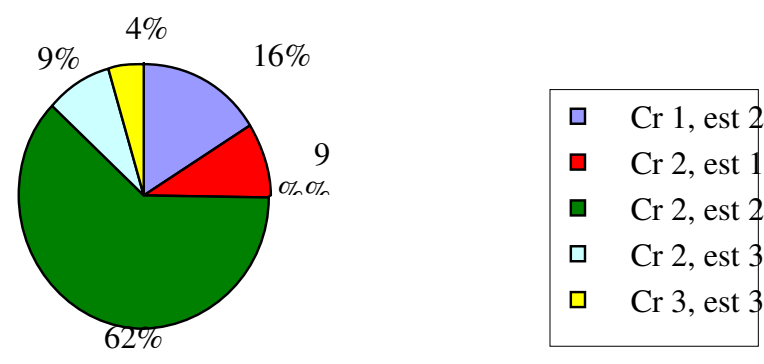

Figura 3: Critérios e estratégias utilizadas pelo Professor 1

\footnotetext{
${ }^{4}$ A função fática está centrada sobre o "contato"(físico ou psicológico). Tudo que numa mensagem serve para estabelecer, manter ou cortar o contato (portanto a comunicação) concerne a essa função. [...] A função fática manifesta essencialmente a necessidade ou o desejo de comunicar. [..] (...)pode-se dizer inclusive que o texto todo funciona como um conjunto organizado de expressões que pouco informam, mas que mantêm os interlocutores em contato. (VANOYE, 1981, p. 54)

V. $4 \mathrm{~N}^{\circ} 1$, Julho, 2006
} 
Percebe-se, na figura 3, que o critério 2 (explicativo) e a estratégia 2 (responsiva), no conjunto de mensagens analisadas, foi a combinação mais utilizada pelo $\mathrm{P} 1$, constituindo $62 \%$ do total das suas mensagens textuais. E, os menos utilizados, foram o critério fático e a estratégia dialógica, com $4 \%$.

Nesse contexto, Moran (1998) diz que "não podemos falar de comunicação de uma forma ideal, mas da comunicação que nos é possível em cada momento com cada pessoa[...]"(p.43). Para este autor, num contexto geral, "a comunicação depende do professor (grifo nosso) e depende do aluno (grifo nosso) e depende também do tipo da relação que estabelece entre nós"(p.43). Falando especificamente da comunicação no ForChat, percebe-se, na Figura 1, que a ênfase é na atitude reflexiva (Critério 1) e interação de caráter responsivo (Estratégia 2), que um depende do outro para que ocorra uma comunicação com encontro de sentidos, para haver interação. Isso quer dizer que, para haver uma comunicação de sentido alunos e professor devem participar das interações, se comunicar, nesse caso, através do ForChat. A interação acontece a partir do "vai e volta", de "perguntas e respostas", tanto do Professor 1 quanto dos alunos.

Apresenta-se, na Figura 4, os critérios e as estratégias utilizadas pelo Professor 2, totalizando 36 mensagens postadas no período de dois meses.

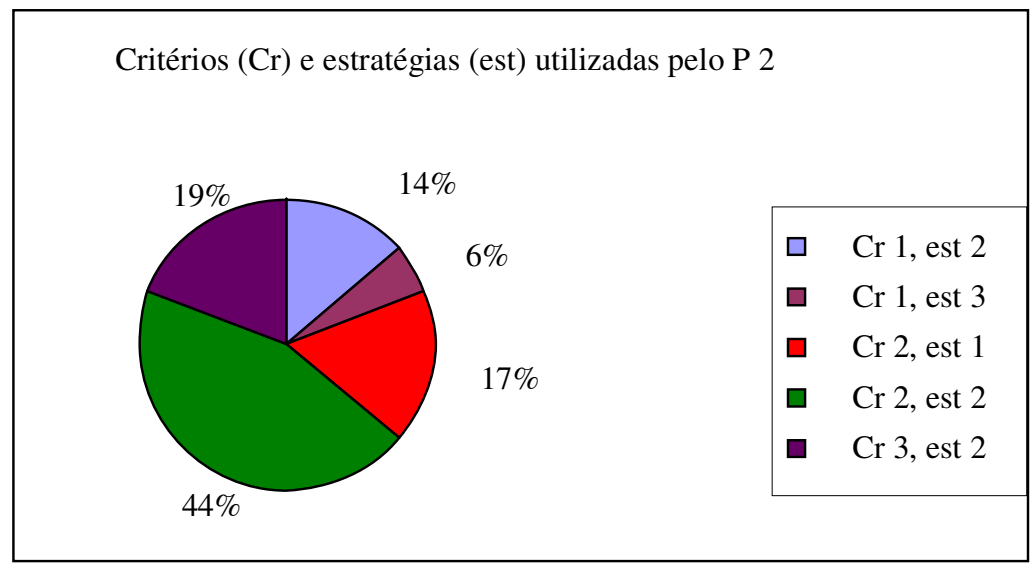

Figura 4: Critérios e estratégias utilizadas pelo Professor 2

Apresenta-se, em destaque na figura 4, o critério 2 (explicativo) e a estratégia 2 (responsiva), do conjunto de mensagens analisadas; esta foi a mais utilizada pelo P 2. Sendo que o critério 1 (cr 1) e a estratégia 2 (est 2) correspondem a $19 \%$ das mensagens textuais postadas pelo $\mathrm{P}$ 2. O critério 3 ( $\mathrm{cr} 3$ ) (fático) e a estratégia 2 (est 2) (responsiva): corresponde a 6\% do total de mensagens textuais postadas pelo $\mathrm{P} 2$.

A Figura 5 representa as combinações dos critérios que identificam as estratégias analisadas no conjunto de mensagens textuais do Professor 3. 


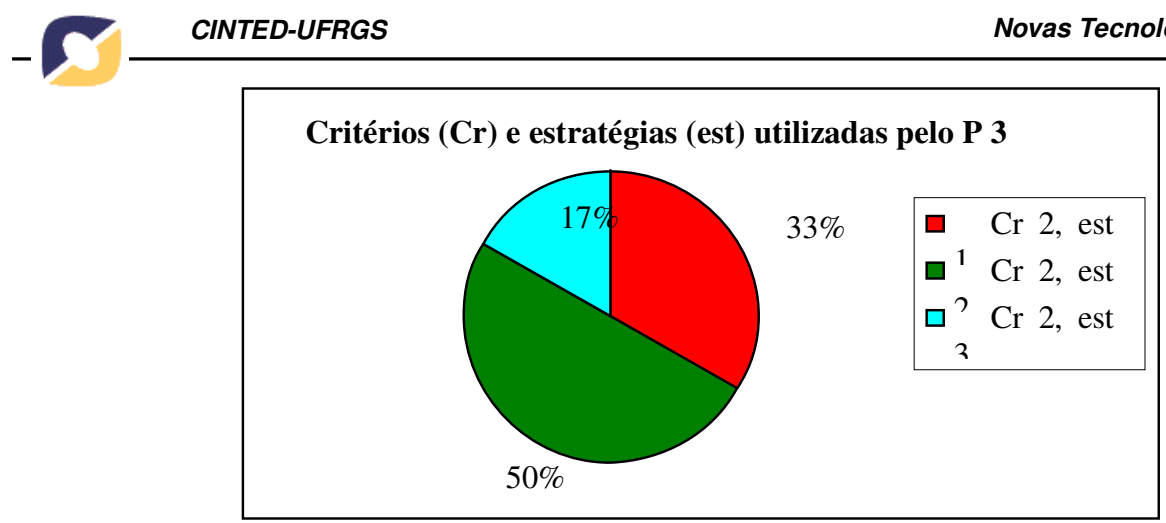

Figura 5: Critérios e estratégias utilizadas pelo Professor 3

$\mathrm{Na}$ figura 5, representando 50\% do total de mensagens, o critério 2 (explicativo) e a estratégia 2 (responsiva) evidenciadas no conjunto de mensagens textuais analisadas foi a mais utilizada pelo P 3. Sendo que o total de mensagens postadas pelo Professor 3 foram 13 mensagens no período de dois meses.

\section{Considerações}

Ao analisar as mensagens textuais de três professores no uso do ForChat, teve-se como objetivo identificar as estratégias utilizadas por eles para a comunicação e interação online com seus alunos. Nesta análise, observou-se que os professores utilizaram a mesma estratégia de comunicação e interação com os alunos na maioria das mensagens textuais postadas para os alunos no ForChat.

Fica-se tentada a dizer que a estratégia de cada professor apresentada no ForChat seguiu os modelos atitudinais/interacionais utilizados presencialmente. A atitude e a estratégia utilizadas pelos professores para manter a comunicação com os alunos do Seminário tanto presencialmente quanto a distância de certo modo se repetiu. No encontro de sentidos, seja em que ambiente for, há necessariamente a construção de outros sentidos e isso se revela de modo diferente em cada sujeito da ação. O sentido produz outros sentidos, ainda que a resposta seja a mesma. Isso permite uma reflexão sobre o fato de que mesmo na repetição há tendência de mudança.

A implicação do professor num ambiente de suporte à aprendizagem on-line faz sentido para ele quando se comunica com os alunos, quando participa, interage, promove discussões e, principalmente, quando se faz presente virtualmente. Isso se pode verificar, numa visão geral, pelo número de mensagens postadas pelos professores (Professor 1; Professor 2; Professor 3), quando estas são equivalentes ao número de postagem de mensagens dos alunos. Desse modo, o professor que mais esteve presente no ForChat foi o que mais promoveu interações, diálogos e participações dos alunos. Observe-se as Figuras 6 e 7, que representam as mensagens postadas pelos alunos e Professores (Professor 1, 2 e 3), no período de dezembro de 2004 a janeiro 2005. 


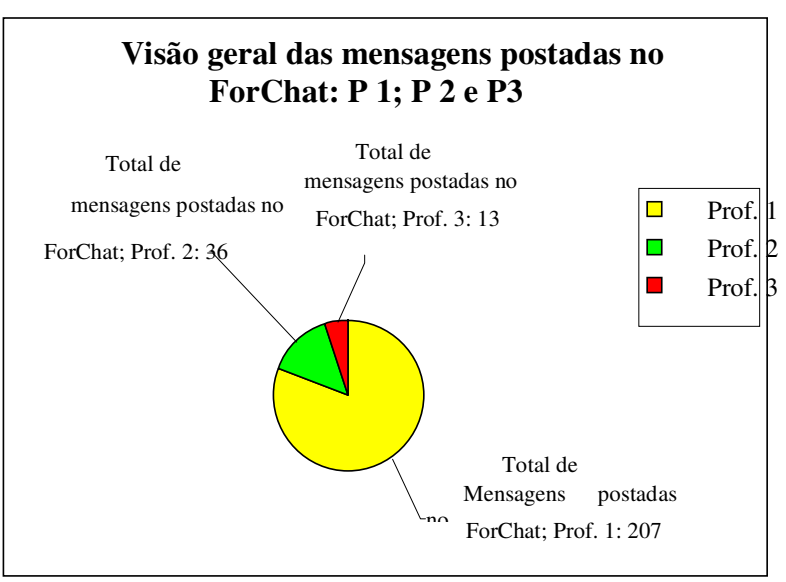

Figura 6: Total de mensagens postadas no ForChat pelos Professores 1, 2 e 3

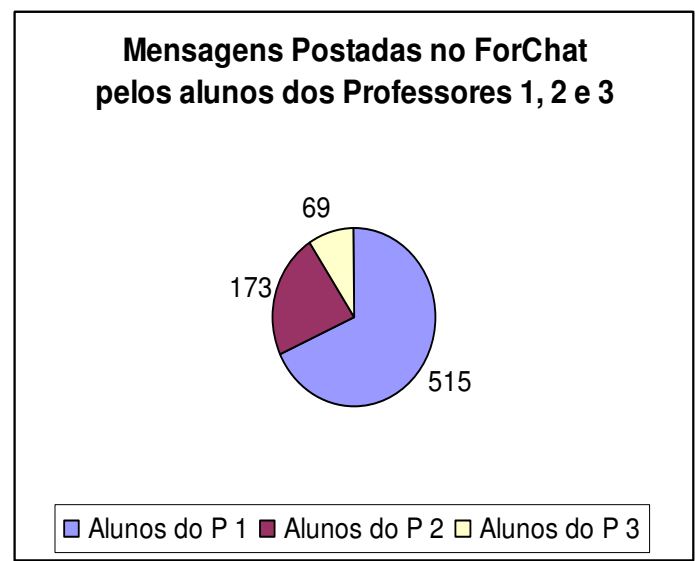

Figura 7: Mensagens postadas no ForChat pelos alunos dos Professores 1, 2 e 3

Como se pode observar, as mensagens postadas pelos professores na Figura 6, ou seja, o P 1 com 207 mensagens; o P 2 com 36 mensagens e o P 3 com 13 mensagens, apresentam uma certa coerência, em relação às mensagens postadas pelos alunos na Figura 7, pois quanto maior a comunicação on-line do professor, maior a interação e participação dos alunos. A esse respeito Moran (1998) afirma que "na comunicação compartilho, troco, interajo - ajo em conjunto com - por meio das palavras[...]. Essa ação provoca uma ação do outro, que se torna re-ação de volta, que confirma ou neutraliza a minha ação. Se eu me sinto confirmado, tenho a tendência a ampliar a interação[...]” (p.19).

Em outras palavras, a comunicação dos professores com os alunos através do ForChat, pôde proporcionar maior interação quando o professor se fez presente, provocando uma reação, uma ação dos alunos para que os mesmos mantivessem uma comunicação, um diálogo efetivo com ele e os demais alunos. Neste contexto, observa-se que, quando o professor dialoga freqüentemente com os alunos, estes participam mais. Ao contrário, aquele professor que não se faz presente com freqüência no ForChat, seus alunos também deixam de participar. Essa observação é confirmada com as palavras de Moran (1998) ao dizer que "se pelo contrário, a cada proposta minha de interação recebo uma resposta desconfirmadora[...] procurarei diminuir as interações com ele, adotarei uma atitude de defesa - não aprofundarei o nível de comunicação[...]"(p.19). A pouca participação ou a falta de comunicação on-line e também a ausência de mensagens textuais dos Professores 2 e 3, durante algumas semanas, no ForChat, pode, como conseqüência, ter levado os alunos a não participar das discussões do Seminário de Projetos Educativos. Nesse contexto, Bakhtin/Voloschinov (1992) afirmariam que "a comunicação verbal, inseparável das outras formas de comunicação, implica conflitos, relações de dominação e de resistência, adaptação ou resistência à hierarquia, utilização da língua pela classe dominante para reforçar seu poder, etc."(p. 14).

A atitude e a estratégia utilizada pelos professores para comunicação com os alunos no ForChat implicou em (re)ações que, em alguns casos, foi uma (re)ação ativa, participativa, e, em outros, de afastamento. No caso do afastamento do professor do ForChat, esse fato pode ter ocasionado uma certa desestabilização no professor. Conforme Valentini et al.5 "[...] um ambiente de aprendizagem concebido com base no paradigma construtivista interacionista

${ }^{5}$ Artigo publicado no site < http://ucsnews.ucs.br/ccha/deps/cbvalent/teorias014/restrito/comunic.html>

$8 \longrightarrow$ V. 4 No 1, Julho, 2006 
leva a desestabilização das concepções tradicionais de ensino e aprendizagem [...]". Axt (1999) complementa ao dizer que o uso de ambientes virtuais "desestabiliza o sistema como um todo"(p. 17).

Mesmo com as diferenças de participação dos Professores 1, 2 e 3, no ForChat, algumas mais efetivas que outras, salienta-se que os indicadores atitudinais (critério explicativo) e interacionistas (estratégia responsiva) foram as que tiveram maior representação no conjunto de mensagens dos professores. Destaca-se, nesse contexto, que viver em diálogo, conforme Emerson (1997), é algo que incentiva seus participantes, mais do que tudo, a cultivar horizontes individualmente construídos, padrões de comportamento, potenciais pessoais - de modo que, seja o que for que nos aconteça numa dada peleja, possamos sempre ter à mão alguma resposta que traga a marca do valor de nossa personalidade (p.322).

Entretanto, é o diálogo, a comunicação, a participação do professor com os alunos que os pode levar a interagir e discutir.

Portanto, a análise do conjunto de mensagens textuais no ForChat, meio de comunicação on-line utilizado pelos três professores, apontou para um professor apenas, por ter ele mantido uma relação de diálogo com os alunos, o qual foi sempre atento a todos os movimentos de diálogo, de discussões de dúvidas, utilizando diferentes critérios e estratégias para que a comunicação entre eles acontecesse com frequiência e intensidade.

Os resultados da análise dos conjuntos de mensagens textuais dos três professores fica como exemplo de como professores podem conduzir um seminário ou uma aula on-line, onde podem ou não provocar as relações de diálogo, podem ou não utilizar o recurso comunicacional on-line para acompanhar e promover a participação de todos os alunos. Constata-se que é a partir da relação de diálogo, da intervenção, da participação efetiva do professor e dos critérios e das estratégias que ele utiliza para manter a comunicação nos ambientes de suporte a aprendizagem on-line, que cada qual pode contribuir para a construção de novos conhecimentos de modo colaborativo.

De fato, os professores que usaram pouco o critério explicativo e a estratégia responsiva no encontro com o ForChat se desestabilizaram num primeiro momento e se reconstruíram ampliando essa estratégia. O Professor 2 se reconstruiu e o Professor 3 apresentou-se mais perturbado, pois foi o que esteve mais ausente no ForChat.

\section{Bibliografia}

ALVES-MAZZOTTI, Alda Judith; GEWANDSZNAJDER, Fernando. O método nas ciências naturais e sociais. Pesquisa quantitativa e qualitativa. São Paulo: Pioneira Thomson. 2. ed. 1999.

AXT, Margarete. Tecnologia na educação, tecnologia para a educação - um texto em construção. Revista Informática na Educação: teoria \& prática, 3(1):51-62, Porto Alegre, UFRGS, 2000.

AXT, Margarete; MARASCHIN, Cleci. Prática pedagógica pensada na indissociabilidade conhecimento-subjetividade. Revista Educação \& Realidade, 22(1):57-80, jan/jun, 1997. 
AXT, Margarete; Medeiros, Fábio; Stuermer-Daitx, Tiago. Desenvolvimento do software de comunicação ForChat. Disponível em $<$ http://www.lelic.ufrgs.br/webteca/for-chat.pdf>, data de acesso: 06/03/2003.

BAKHTIN, Mikhail. Estética da Criação Verbal. $3^{\mathrm{a}}$. ed., São Paulo: Martins Fontes, 2000.

BAKHTIN, Mikhail(Volochinov). Marxismo e filosofia da linguagem. $6^{\text {a }}$ ed. São Paulo: Hucitec, 1992.

COSCARELLI, Carla Viana(Organizadora). Novas tecnologias, novos textos, novas formas de pensar. Belo Horizonte: Autêntica, 2002.

EMERSON, Caryl. Os 100 primeiros anos de Mikhail Bakhtin; tradução Pedro Jorgensen Jr. - Rio de Janeiro: DIFEL, 2003.

FIORENTINI, Leda Maria Rangearo; MORAES, Raquel de Almeida (orgs.) Linguagens e interatividade na educação a distância. Rio de Janeiro: DP\&A, 2003.

LEDUR, Rejane Reckziegel. Professores de Arte e Arte Contemporânea: Contextos de Produção de Sentido. Dissertação de Mestrado. PPGE, UFRGS. Porto Alegre, 2005.

MEHLECKE, Querte T.C.; AXT, Margarete; TAROUCO, Liane M.R. For-Chat: uma comunidade virtual construindo sentido, autoria e conceitos através do discurso em um ambiente cooperativamente interativo. Artigo publicado no I Ciclo de Palestras sobre Novas Tecnologias na Educação - CINTED 2003. Disponível em: $<\mathrm{http}: / /$ www.cinted.ufrgs.br/eventos/cicloartigosfev2003/ palestras03index.htm> Acesso em: 20/03/2004.

MEHLECKE, Querte T.C.; TAROUCO, Liane M.R. ESTUDANDO AMBIENTES DE SUPORTE PARA EDUCAÇÃO A DISTÂNCIA: Uma mediação para aprendizagem cooperativa online. Artigo publicado no I Ciclo de Palestras sobre Novas Tecnologias na Educação- $\quad$ CINTED 2003. Disponível em <http://www.cinted.ufrgs.br/eventos/cicloartigosfev2003/ palestras03index.html> Acesso em: 10/09/2004

MEHLECKE, Querte; PEREIRA, Alda; TAROUCO, Liane. Estratégias de interação entre tutor e estudantes em Educação a Distância. Artigo publicado no I Ciclo de Palestras sobre Novas Tecnologias na Educação- CINTED 2005. Disponível em $<$ http://www.cinted.ufrgs.br/renote/maio2005/apresentacoes/apa27_interacao_querte.pd f> Acesso em: 10/05/2006.

2 MOREIRA, Gleice Maria de Oliveira. Tecnologias da Informação e Comunicação na Escola Pública: Sentidos produzidos na formação continuada de professores. PPGE,UFRGS. Porto Alegre, 2005.

MORAN, José Manuel. Contribuições para uma pedagogia da educação on-line. In Marco Silva(org) Educação On-line. Teorias, práticas, legislação e formação corporativa. São Paulo: edições Loyola, 2003.Pg 39-50

MORAN, José Manuel. Mudar a forma de ensinar e aprender com tecnologias. Revista Interações, Vol. 5 - No 9 - pp. 57-72. Jan/Jun 2000a. 
MORAN, José Manuel. Educação inovadora em Ambientes Presenciais e Virtuais. Mackenzie Educação, Arte e História da Cultura, ano 1, nº, 2001.

3 MORAN, José Manuel. Novas Tecnologias e o Reencantamento do Mundo. Revista Tecnologia Educacional. Rio de Janeiro, vol. 23, n 126, setembro-outubro, 1995, p. 24-26.

PETERS, Otto. Didática do Ensino a Distância. São Leopoldo/RS : Unisinos, 2001.

2002.

A educação a distância em transição. São Leopoldo/RS: UNISINOS,

SOUZA, Elmara Pereira. Tecnologias digitais na escola pública: possibilidade de exercício de autoria na formação de professores. PPGIE, UFRGS. Porto Alegre, 2005.

SOUZA, Geraldo Tadeu. Introdução à teoria do enunciado concreto do círculo Bakhtin/Voloshinov/Medvedev. São Paulo: Humanitas, 2002.

TAROUCO, Liane M.R. Aprendizagem Colaborativa. I Esud - Petrópolis, Rio de Janeiro, 26 a 28 de março de 2002. Disponível em: $<$ http://www.sead.ufrj.br/esud/material/Liane_Tarouco_ufrgsesud.ppt $>$ Acesso em: 20/07/2004. 